\title{
Helicobacter pylori hopQ alleles (type I and II) in gastric cancer
}

\author{
HAMED EBRAHIMZADEH LEYLABADLO ${ }^{1}$, MINA YEKANI ${ }^{2}$ and REZA GHOTASLOU ${ }^{1,2}$ \\ ${ }^{1}$ Immunology Research Center, Tabriz University of Medical Sciences, Tabriz 51636-39888; \\ ${ }^{2}$ Department of Microbiology, School of Medicine, Tabriz University of Medical Sciences, Tabriz 51664-14766, Iran
}

Received December 22, 2015; Accepted March 2, 2016

DOI: $10.3892 /$ br.2016.634

\begin{abstract}
The Helicobacter pylori (H.pylori) outer membrane protein (HopQ) of is one of the proteins involved in bacterial adherence to gastric mucosa and has been suggested to have a role in the virulence of $H$. pylori. The aim of the present study was to determine the association between $H$. pylori virulence types I and II hop $Q$ genotypes and patients with different gastrointestinal diseases. A polymerase chain reaction-based assay was used to determine the presence of type I and type II hop $Q$ genes in $88 \mathrm{H}$. pylori strains isolated from $H$. pylori-infected patients. Of the total $88 \mathrm{H}$. pylori isolates, type I and type II hop $Q$ alleles were detected in $52(59.1 \%)$ and $36(40.9 \%)$, respectively. A significant association was found between type I hop $Q$ gene and gastric cancer [odds ratio, 2.3; 95\% confidence interval $(\mathrm{CI}), 1.3-4.1]$ and gastric ulcers (odds ratio, 2.5; 95\% CI, 1.4-4.3). A significant association was also identified between the type II hop $Q$ gene and gastric cancer (odds ratio, 2.4; 95\% CI, 1.1-3.0). The association between $h o p Q$ type I and hop $Q$ type II genotypes and clinical status suggest that these genes may be helpful in the universal prediction of specific disease risks.
\end{abstract}

\section{Introduction}

Helicobacter pylori (H.pylori) is a Gram-negative bacterium that persistently colonizes in the stomachs of more than half the human population (1). Colonization of the stomach by $H$. pylori consistently induces gastric inflammation, known as superficial chronic gastritis, and is a risk factor for the development of peptic ulcer disease and gastric malignancies (2). H. pylori strains are thought to possess various virulence factors, which contribute to digestive disease complications (3). In 2005, it was reported that $H$. pylori hop $Q$ genotypes are associated with an increased risk for peptic ulcer disease (4). $H$. pylori genomes contain $\sim 30$ paralogous hop genes, which

Correspondence to: Professor Reza Ghotaslou, Immunology Research Center, Tabriz University of Medical Sciences, No. 11 Building, Daneshgah Street, Tabriz 51636-39888, Iran E-mail: rzgottaslo@yahoo.com

Key words: Helicobacter pylori, virulence factor, outer membrane proteins encode outer membrane proteins (5). HopQ is localized at the surface of $H$. pylori (6). The H.pylori outer membrane protein (Hop) family is the largest and includes adhesions such as BabA (HopS) (7), SabA (HopP) (8), OipA (HopH) (9), AlpAB (HopB and HopC) (10) and HopQ (11). However, nothing is known regarding the functional properties of HopQ and this study did not provide further data regarding disease-specific virulence factor of hop $Q$. H. pylori hop $Q$ alleles exhibit a high level of genetic diversity, and two families of hop $Q$ alleles have been described (type I hop $Q$ and type II $h o p Q$ ) (12). Iran is a developing country with a high prevalence of $H$. pylori infections, among symptomatic and asymptomatic individuals, and the prevalence is $\mathbf{5 9 0 \%}$ in the northwestern part of the country (13). The aim of the present study was to determine the association between $H$. pylori virulence type I and type II hop $Q$ genotypes and patients with different gastrointestinal diseases.

\section{Materials and methods}

Patients. In the study, patients undergoing upper gastric endoscopy due to different digestive diseases that visited the hospitals in Tabriz (Iran) were included. The standard number of gastric biopsy samples for patients who were suspected of being infected with $H$. pylori was obtained for routine culture and histological investigations. Patient groups according to endoscopic and pathology findings were: Gastric ulcer (GU), duodenal ulcer (DU), gastritis and gastric carcinoma (GC). Written informed consent was obtained from all the patients prior to entering the study, and the study was approved by the Regional Ethics Committee, Tabriz University of Medical Sciences (Tabriz, Iran; no. 5/47/1375, April 4, 2015).

H. pylori isolates and bacterial culture. The presence of H. pylori was determined by cultures placed on Brucella agar (Merck KGaA, Darmstadt, Germany) containing 5\% sheep blood and antibiotics such as amphotericin B $(5 \mathrm{mg} / \mathrm{ml})$, trimethoprim lactate $(5 \mathrm{mg} / \mathrm{l})$, vancomycin $(10 \mathrm{mg} / \mathrm{ml})$ and polymixin-B $(2,500 \mathrm{U} / \mathrm{ml})$. Plates were incubated at $37^{\circ} \mathrm{C}$, in $10 \% \mathrm{CO}_{2}$ conditions, and were subsequently identified as H. pylori, based on: Colony morphology, Gram-staining and positive oxidase, catalase and urease tests. In the present study, the H.pylori American Type Culture Collection (ATCC) 43504 strain (ATCC, Manassas, VA, USA) was used as a reference.

DNA extraction and polymerase chain reaction (PCR). Bacterial DNA was extracted from single colonies of $H$. pylori 
Table I. Amplification primers for amplification used in the study.

\begin{tabular}{lcclc}
\hline Study, year & DNA region amplified & Primers & \multicolumn{1}{c}{ Primer sequence } & Refs. \\
\hline Kidd et $a l, 1999$ & glm $\mathrm{M}$ & HP-F & AAGCTTTTAGGGGTGTTAGGGGTTT & $(16)$ \\
& & HP-R & AAGCTTACTTTCTAACACTAACGC & \\
Sicinschi et al, 2012 & Type I $h o p Q$ & HP-F & CAACGATAATGGCACAAACT & $(17)$ \\
& & HP-R & GTCGTATCAATAACAGAAGTTG & $(17)$ \\
Sicinschi et al, 2012 & Type II $h o p Q$ & HP-F & TCCAATCCAGAAGCGATTAA & \\
& & HP-R & GTTTTAATGGTTACTTCCACC & \\
& & & &
\end{tabular}

Table II. Distribution of Helibacter pylori hopQ genes (type I and II) among the different groups.

\begin{tabular}{lcccc}
\hline Diseases (no.) & Rate of type I hopQ, no. (\%) & Odd ratio (95\% CI) & Rate of type II hop $Q$, no. (\%) & Odds ratio (95\% CI) \\
\hline Gastric carcinoma (26) & $18(69.2)$ & $2.3(1.3-4.1)$ & $16(61.5)$ & $2.4(1.1-3.0)$ \\
Duodenal ulcer (16) & $6(37.5)$ & $0.6(0.3-1.0)$ & $2(12.5)$ & $0.2(0.1-0.4)$ \\
Gastric ulcer (10) & $10(100.0)$ & $2.5(1.4-4.3)$ & $4(40.0)$ & $0.7(0.4-1.2)$ \\
Gastritis (36) & $18(50.0)$ & Control group & $14(38.9)$ & Control group
\end{tabular}

HopQ, Helibacter pylori outer membrane protein; CI, confidence interval.

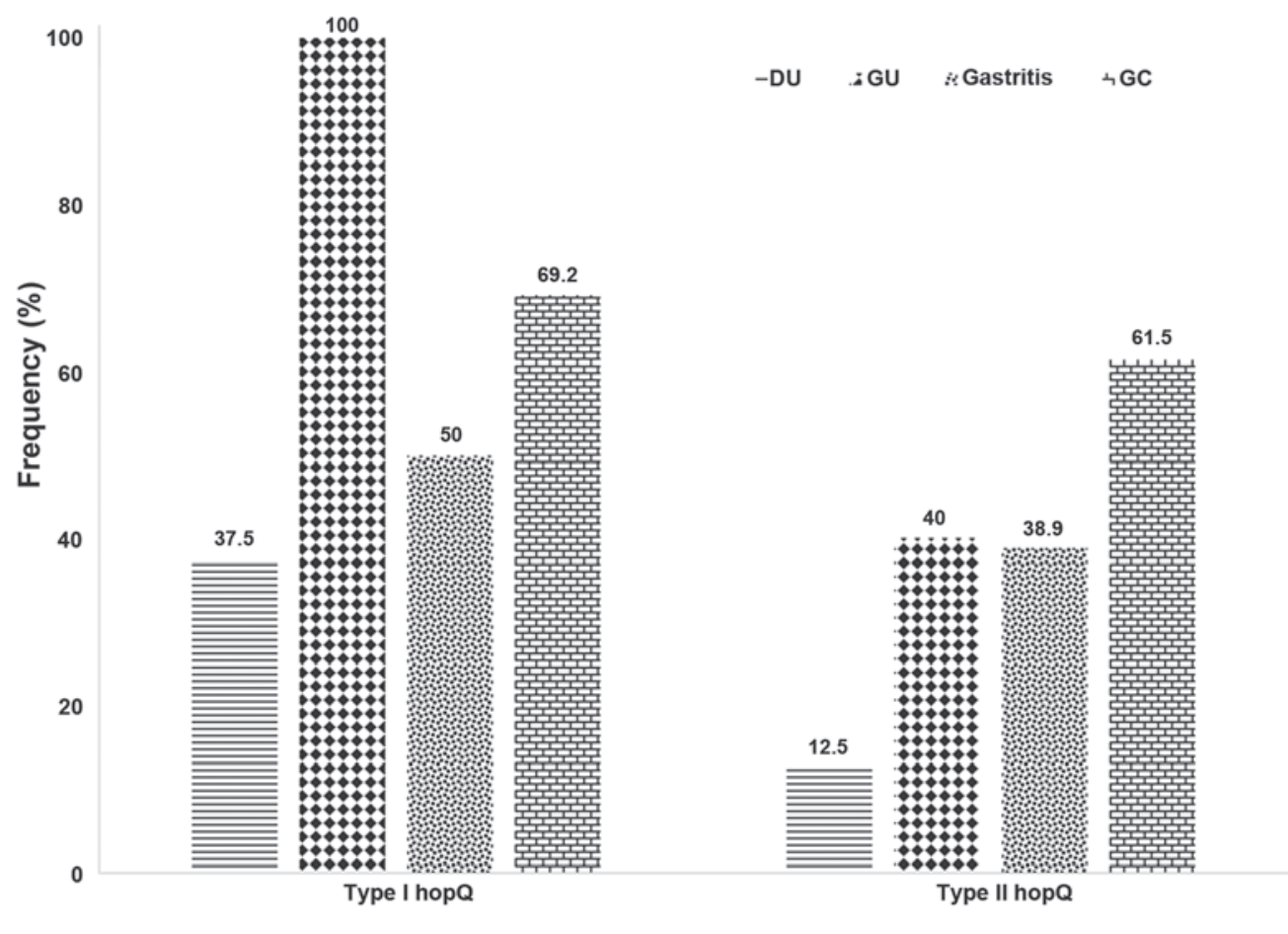

Figure 1. Frequency of hopQ types among different diseases.

and their DNA was extracted by sodium dodecyl sulphate, proteinase $\mathrm{K}$ and the cetyltrimethyl ammonium bromide method (14) and stored at $-20^{\circ} \mathrm{C}$. Initially, the PCR assay was used to detect the H. pylori-specific ureC ( $g l m \mathrm{M})$ gene for confirmation of $H$. pylori strains (15). In this experiment, hop $Q$ type I and type II genes were detected by the PCR method $(16,17)$ (Table I), under the following conditions for both genes: 35 cycles at $94^{\circ} \mathrm{C}$ for $60 \mathrm{sec}$, at $54^{\circ} \mathrm{C}$ for $45 \mathrm{sec}$, and at $72^{\circ} \mathrm{C}$ for $65 \mathrm{sec}$, and a final extension at $72^{\circ} \mathrm{C}$ for $10 \mathrm{~min}$. PCR products were analyzed on $1.5 \%$ agarose and the strains containing the hop $Q$ type I and hop $Q$ type II genes were used as a positive control.

Statistical analysis. The data obtained were analyzed by SPSS (version 19; IBM, Corp., Armonk, NY, USA) and the $\chi^{2}$ test and Fisher's exact test was used to compare the clinical 
outcomes and the presence of genes. $\mathrm{P}<0.05$ was considered to indicate a statistically significant difference.

\section{Results}

H. pylori culture positive. From 286 gastric biopsies, $88(30.76 \%)$ were positive for $H$. pylori. Of the patients (including $88 \mathrm{H}$. pylori-positive) that attended the endoscopy ward, 6 suffered from anemia (6.8\%), 24 from epigastria pain (27.3\%), 10 from gastro-esophageal reflux disease $(11.4 \%)$, 46 from dyspepsia (52.3\%), and 2 from gastrointestinal bleeding (2.3\%). According to endoscopy and pathology findings, among the remaining $88 \mathrm{H}$. pylori culture-positive patients, 10 (11.4\%) had GU, $16(18.2 \%)$ had DU, $36(41.0 \%)$ had gastritis and 26 (29.5\%) had GC (Fig. 1).

Using primers for the ure $\mathrm{C}(\mathrm{g} \operatorname{lm} \mathrm{M})$ gene, the $\mathrm{PCR}$ product of 294-base pairs was obtained in all 88 strains. The mean age of patients was $40 \pm 15$ years, ranging from 18 to 84 years and including $40(45.5 \%)$ males and $48(54.5 \%)$ females. The data showed that of 88 isolates, hop $Q$ type I was present in $52(59.1 \%)$ isolates and hop $Q$ type II was found in $36(40.9 \%)$ isolates. Table II depicts the prevalence of these genes in association with the different disease groups.

Associations between hopQ types and disease. There were no statistically significant associations between age and gender with hop $Q$ types $(\mathrm{P}>0.05)$. Statistical analysis indicated significant associations between hop $Q$ type I and GC [odds ratio, 2.3; 95\% confidence interval (CI), 1.3-4.1] and GU (odds ratio, $2.5 ; 95 \% \mathrm{CI}, 1.4-4.3)$. Type II hop $Q$ was also found to be significantly predominant in patients with gastric cancer (odds ratio, 2.4; 95\% CI, 1.1-3.0). A combination of hopQ type I/hop $Q$ type II genotypes were observed in 36 (40.9\%) patients and statistical analysis demonstrates that there is a significant association between the simultaneous presence of these two genes $(\mathrm{P}=0.004)$.

\section{Discussion}

$H$. pylori cause the most prevalent bacterial infections globally (18). The adherence of $H$. pylori to the gastric mucosa have important roles in the initial colonization and long-term persistence on the gastric mucosa as well as in the intensity of the resulting inflammatory response. Colonization of $H$. pylori usually does not result in clinical consequences but can increase the risk of developing peptic ulcer diseases, gastric adenocarcinoma and lymphoma (19). It has been suggested that specific genotyping-based analysis of $H$. pylori isolates can be useful for predicting post-infection disorders (20).

In the present study, the prevalence of $H$. pylori hop $Q$ type I and type II genotypes was analyzed in patients with different gastrointestinal diseases. The predominant genotype In the Azerbaijan area was the hop $Q$ type I genotype found in $52(59 \%)$ cases and this high rate of hop $Q$ type I was in contrast to a study, also performed in Iran, that considered the finding of 52\% hop $Q$ type II genotypes as being high (21). However, the present finding concerning the frequency of hop $Q$ type I is consistent with a previous study conducted in the USA (11). Geographical differences in the distribution of type I and type II hopQ alleles have been noted. For example, the majority of $H$. pylori strains isolated in East Asia are cag PAI-positive and contain type I hop $Q$ alleles. Type II alleles are commonly identified in $H$. pylori strains isolated in Western countries, but are uncommon among $H$.pylori strains from East Asia (4).

In the present study, the rate of the hop $Q$ type II genotype was found most commonly in GC (61.5\%). This finding is similar to results obtained in the North of Iran (21). The present findings showed that hop $Q$ type I was most predominant in patients with $\mathrm{GU}$, which is in disagreement with the study conducted in the North of Iran (21). To the best of our knowledge, this is the first study on the prevalence of $H$.pylori hop $Q$ alleles, among patient with gastrointestinal disease in the Azerbaijan area, and this is the second study in Iran to determine the prevalence and association of these virulence genes with clinical outcomes. The present findings have shown that the hop $Q$ type I and type II genotypes are associated with gastric cancer. The study by Cao and Cover (11) was conducted in the USA and reported that there was a significant association between the carriers of $H$. pylori hop $Q$ type I among the peptic ulcer patients (11). However, Ohno et al (22) did not identify an association between the two hopQ alleles and clinical outcomes. Additionally, the combination of hop $Q$ type I/hop $Q$ type II genotypes were evaluated in the present study in association with the clinical outcomes and statistically significant correlation was identified between these alleles and disease conditions. It appears that these two genes are located in one place and may have a synergistic effect.

The prevalence and association between clinical outcome and the $H$. pylori virulence gene was reported to be different among various countries, regions, ethnicities and patient groups. The differences between studies may be due to geographical area, sample size, studied groups, ethnicity, primer sets, PCR conditions and the variety of bacterial strains. The weakness and limitations of the study firstly were low sample size and the small number of studies performed to obtain a whole evaluation on the prevalence of genes.

If additional studies showed similar results to the present findings, this may lead to improved diagnostic policies for clinicians to fight the present doctrine for gastric diseases. In conclusion, the association between hop $Q$ types I and II, and gastric cancer outcomes, may suggest that these genes may be helpful for the prediction of specific-disease risk.

\section{Acknowledgements}

The authors are grateful to Dr H. Banazadeh Baghi for the English editing. The study was financially supported by Tabriz University of Medical Sciences.

\section{References}

1. Cover TL and Blaser MJ: Helicobacter pylori in health and disease. Gastroenterology 136: 1863-1873, 2009.

2. Cover TL, Berg DE, Blaser MJ and Mobley H: Helicobacter pylori pathogenesis. In: Principles of bacterial pathogenesis Academic Press, San Diego, CA, pp510-558, 2001.

3. Costa AC, Figueiredo C and Touati E: Pathogenesis of Helicobacter pylori infection. Helicobacter 14 (Suppl 1): 15-20, 2009.

4. Cao P, Lee KJ, Blaser MJ and Cover TL: Analysis of hop $Q$ alleles in East Asian and Western strains of Helicobacter pylori. FEMS Microbiol Lett 251: 37-43, 2005. 
5. Alm RA, Bina J, Andrews BM, Doig P, Hancock RE and Trust TJ: Comparative genomics of Helicobacter pylori: Analysis of the outer membrane protein families. Infect Immun 68: 4155-4168, 2000.

6. Sabarth N, Hurvitz R, Schmidt M, Zimny-Arndt U, Jungblut PR, Meyer TF and Bumann D: Identification of Helicobacter pylori surface proteins by selective proteinase $\mathrm{K}$ digestion and antibody phage display. J Microbiol Methods 62: 345-349, 2005.

7. Ilver D, Arnqvist A, Ögren J, Frick IM, Kersulyte D, Incecik ET, Berg DE, Covacci A, Engstrand L and Borén T: Helicobacter pylori adhesin binding fucosylated histo-blood group antigens revealed by retagging. Science 279: 373-377, 1998.

8. Mahdavi J, Sondén B, Hurtig M, Olfat FO, Forsberg L, Roche N, Angstrom J, Larsson T, Teneberg S, Karlsson KA, et al: Helicobacter pylori SabA adhesin in persistent infection and chronic inflammation. Science 297: 573-578, 2002.

9. Yamaoka Y, Kwon DH and Graham DY: A M(r) 34,000 proinflammatory outer membrane protein (oipA) of Helicobacter pylori. Proc Natl Acad Sci USA 97: 7533-7538, 2000.

10. Odenbreit S, Till M, Hofreuter D, Faller G and Haas R: Genetic and functional characterization of the alpAB gene locus essential for the adhesion of Helicobacter pylori to human gastric tissue. Mol Microbiol 31: 1537-1548, 1999.

11. Cao P and Cover TL: Two different families of $h o p Q$ alleles in Helicobacter pylori. J Clin Microbiol 40: 4504-4511, 2002.

12. Backert S and Selbach M: Role of type IV secretion in Helicobacter pylori pathogenesis. Cell Microbiol 10: 1573-1581, 2008.

13. Ghotaslou R, Milani M, Akhi MT, Nahaei MR, Hasani A, Hejazi MS and Meshkini M: Diversity of Helicobacter pylori caga and vaca genes and its relationship with clinical outcomes in Azerbaijan, Iran. Adv Pharm Bull 3: 57-62, 2013.
14. Sambrook J and Russell DW: Molecular cloning. A laboratory manual. 3rd edition. Cold Spring Harbor Laboratory Press, New York, NY, 2001.

15. Espinoza MGC, Vazquez RG, Mendez IM, Vargas CR and Cerezo SG: Detection of the glmM gene in Helicobacter pylori isolates with a novel primer by PCR. J Clin Microbiol 49: 1650-1652, 2011

16. Kidd M, Louw JA and Marks IN: Helicobacter pylori in Africa: Observations on an 'enigma within an enigma'. J Gastroenterol Hepatol 14: 851-858, 1999.

17. Sicinschi LA, Correa P, Bravo LE, Peek RM Jr, Wilson KT, Loh JT, Yepez MC, Gold BD, Thompson DT, Cover TL, et al: Non-invasive genotyping of Helicobacter pylori cagA, vacA, and $h o p Q$ from asymptomatic children. Helicobacter 17: 96-106, 2012.

18. Rafeey M, Ghotaslou R, Nikvash S and Hafez AA: Primary resistance in Helicobacter pylori isolated in children from Iran. J Infect Chemother 13: 291-295, 2007.

19. Ghotaslou R, Leylabadlo HE and Asl YM: Prevalence of antibiotic resistance in Helicobacter pylori: A recent literature review. World J Methodol 5: 164-174, 2015.

20. Graham DY and Fischbach L: Helicobacter pylori infection. N Engl J Med 363: 595-596, author reply 596, 2010.

21. Talebi Bezmin Abadi A and Mohabbati Mobarez A: High prevalence of Helicobacter pylori hop $Q$ II genotype Isolated from Iranian patients with gastroduodenal disorders. J Pathogens 2014: 842469, 2014.

22. Ohno T, Sugimoto M, Nagashima A, Ogiwara H, Vilaichone RK, Mahachai V, Graham DY and Yamaoka Y: Relationship between Helicobacter pylori hop $Q$ genotype and clinical outcome in Asian and Western populations. J Gastroenterol Hepatol 24: 462-468, 2009 